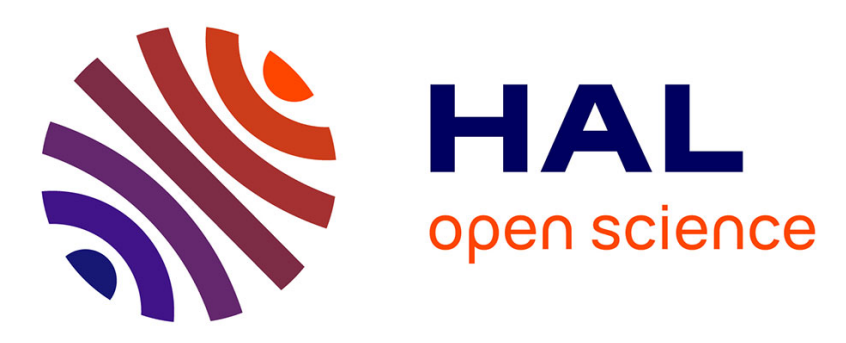

\title{
Trapping and exclusion zones in complex streaming patterns around a large assembly of microuidic bubbles under ultrasound
}

Thomas A Combriat, Flore Mekki-Berrada, Pierre Thibault, Philippe Marmottant

\section{- To cite this version:}

Thomas A Combriat, Flore Mekki-Berrada, Pierre Thibault, Philippe Marmottant. Trapping and exclusion zones in complex streaming patterns around a large assembly of microuidic bubbles under ultrasound. Physical Review Fluids, 2018, 3 (1), pp.013602. 10.1103/PhysRevFluids.3.013602 . hal$01709188 \mathrm{v} 2$

\section{HAL Id: hal-01709188 \\ https://hal.science/hal-01709188v2}

Submitted on 8 Jul 2019

HAL is a multi-disciplinary open access archive for the deposit and dissemination of scientific research documents, whether they are published or not. The documents may come from teaching and research institutions in France or abroad, or from public or private research centers.
L'archive ouverte pluridisciplinaire HAL, est destinée au dépôt et à la diffusion de documents scientifiques de niveau recherche, publiés ou non, émanant des établissements d'enseignement et de recherche français ou étrangers, des laboratoires publics ou privés. 


\title{
Trapping and exclusion zones in complex streaming patterns around a large assembly of microfluidic bubbles under ultrasound
}

\author{
Thomas Combriat, ${ }^{*}$ Flore Mekki-Berrada, Pierre Thibault, and Philippe Marmottant ${ }^{\dagger}$ \\ CNRS / Université Grenoble-Alpes, LIPhy UMR 5588, Grenoble, F-38401, France
}

(Received 20 September 2017; published 11 January 2018)

\begin{abstract}
Pulsating bubbles have proved to be a versatile tool for trapping and sorting particles. In this article, we investigate the different streaming patterns that can be obtained with a group of bubbles in a confined geometry under ultrasound. In the presence of an external flow strong enough to oppose the streaming velocities but not drag the trapped bubbles, we observe either the appearance of exclusion zones near the bubbles or asymmetric streaming patterns that we interpret as the superposition of a two-dimensional (2D) streaming function and of a potential flow. When studying a lattice of several bubbles, we show that the streaming pattern can be accurately predicted by superimposing the contributions of every pair of bubbles present in the lattice, thus allowing one to predict the sizes and the shapes of exclusion zones created by a group of bubbles under acoustic excitation. We suggest that such systems could be used to enhance mixing at a small scale or to catch and release chemical species initially trapped in vortices created around bubble pairs.
\end{abstract}

DOI: 10.1103/PhysRevFluids.3.013602

\section{INTRODUCTION}

Microfluidic devices play growing roles in chemical, biological, and physical applications since they allow the observation and manipulation of micron-scale objects such as cells, particles, droplets, and chemicals in small-volume samples. The combination of microfluidics with acoustics has recently led to the development of new applications such as acoustic tweezers and localized acoustic streaming flows [1-3]. At these small scales, acoustic streaming results from the interaction of high-frequency sound waves $(>10 \mathrm{MHz})$ with the channel boundaries, leading to steady flows. It is known that acoustic streaming can also develop at the surface of inclusions such as solid microparticles or bubbles [4]. In the case of oscillating bubbles, acoustic streaming is particularly intense when the acoustic excitation matches the mechanical resonance of the bubbles (typically $100 \mathrm{kHz}$ for $\phi 60 \mu \mathrm{m}$ bubbles), leading both to large-amplitude pulsations and steady flows around bubbles $[5,6]$. This phenomenon therefore holds great potential for various applications including mixing of liquids or reactants, provided a method can be employed to produce and hold bubbles on specified locations [7].

It was recently found that microbubbles could be held in specific locations even under flow, while still vibrating. Bubbles are simply trapped on micropits placed on one wall of the microchannel [8]; under these circumstances, when a couple of bubbles are excited together, oscillatory and translational modes of adjacent bubbles can combine, resulting in strong dipolar flow fields $[6,9,10]$. The aim of this paper is twofold: (i) to investigate the complex flows resulting from the superposition of an ensemble of many pulsating bubbles and (ii) to understand the origin and the role of closed recirculation zones observed in the presence of an incoming flow.

\footnotetext{
*Thomas.Combriat@univ-grenoble-alpes.fr

thttp://www-liphy.ujf-grenoble.fr/equipe/dyfcom/marmottant
} 
Here we develop a setup designed to hold microfluidic bubbles at a specific position and to expose them to ultrasound. We then characterize the streaming pattern around a pair of bubbles under external flow. A prediction for the streamlines is proposed. Finally, we explore the validity of this approach to a group of bubbles.

\section{MATERIAL AND METHODS}

Bubbles are produced and observed inside a microfluidic channel; see Fig. 1 for a sketch of the experimental setup. The microfluidic chip is produced using standard soft lithography techniques [11]. Two polydimethylsiloxane (PDMS, Sylgard 184, Dow Corning) layers were sealed together, one molded with a flow-focusing junction connected to a straight observation channel ( $2 \mathrm{~mm}$ wide, $25 \mu \mathrm{m}$ high), one molded with micropits ( $40 \mu \mathrm{m}$ in diameter, $40 \mu \mathrm{m}$ in depth). The flow-focusing junction is fed with gaseous nitrogen and deionized water containing $5 \% \mathrm{w} / \mathrm{w}$ of surfactant (commercial Dreft dish-washing liquid) with flow controlled by a syringe pump (Harvard Apparatus), in order to generate monodisperse bubbles with radii between 20 and $50 \mu \mathrm{m}$. Since the bubble diameters are larger than the channel height, they are squeezed between the upper and lower walls, adopting pancake-like shapes, as sketched in Fig. 1(b).

The acoustic excitation is a plane wave, produced by a $100-\mathrm{kHz}-$ centered transducer (Ultran), partially immersed in a tank of approximate dimension $20 \times 10 \times 5 \mathrm{~cm}^{3}$ filled with deionized water. The microchannel is fixed on the bottom of this tank, in the acoustic field of the transducer, taking advantage of the natural adhesion between the tank wall and the PDMS [see Fig. 1(a)]. In a typical experiment, bubbles are first produced from the flow-focusing outlet at a prerequisite size and are then dragged by the main flow. When bubbles occasionally flow over micropits, they tend to be captured and can later be released by increasing the flow velocity. This technique enables studying the bubbles at rest over long periods of time, while sound can be switched on or off and the frequency tuned until it matches the bubble resonance frequency (also called Minnaert's frequency).

Quantitative studies are then performed using video analyses. The videos are recorded using a fast Phantom V2511 camera, mounted on an Olympus IX73 inverted microscope. Flows are visualized using tracer particles $(2 \mu \mathrm{m}$ diameter), which can be tracked to reconstruct trajectories using a homemade particle tracking software [12]. The external flow velocity $U_{\infty}$ was measured by following different tracers far upstream from the bubble pair.

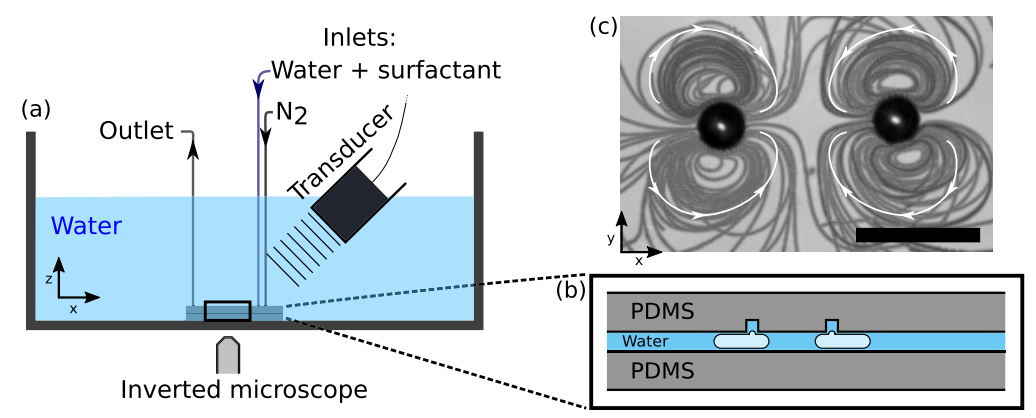

FIG. 1. (a) Scheme of the experimental setup showing the water tank, the microfluidic device where the bubbles are created and studied, the external acoustic excitation, and the microscope used for visualization. (b) Side-view detail showing the PDMS microchannel, with two bubbles trapped on micropits imprinted in the upper wall. (c) Streaming fountain pattern around two vibrating bubbles without external flow. In this experiment, the distance between the two bubbles is $150 \mu \mathrm{m}$, the scale bar is $100 \mu \mathrm{m}$, and the excitation frequency is $91 \mathrm{kHz}$. The flow is fed with $2-\mu \mathrm{m}$ tracers in order to visualize the trajectories. Arrows indicate the direction of motion of the particles. 


\section{INTERACTION BETWEEN THE STREAMING AND AN EXTERNAL FLOW}

\section{A. Exclusion zone created by two-bubble streaming flows}

\section{Experimental results: Emergence of a ionosphere-like exclusion zone}

Figure 1(c) shows the in-plane streaming pattern obtained around two acoustically excited bubbles in a surrounding liquid at rest. This pattern consists of a four-vortice structure, with vorticity signs corresponding to the fountain pattern observed by Rallabandi et al. [6] in the case of a single bubble in contact with a wall. As discussed in Ref. [9], the presence of these vortices is induced by the interaction of the two bubbles. The intensity and the direction of the flow velocity in these vortices depend on the excitation frequency and the ratio between bubbles radii and their relative distance.

When an external flow is applied along the bubble pair direction, one can see the emergence of four stagnation points [see Fig. 2(a)]. On these locations, the total velocity of the flow is vanishing, with the streaming velocity and the external velocity canceling each other. Two of these points are located along the bubble pair axis (one upstream from the bubble pair, one in between the two bubbles) and the two others on the side of the downstream bubble. These points are stable along one axis but unstable in the orthogonal axis, a typical feature of saddle points. This delimits four vortices [see Fig. 2(a)] with extensions that decrease when the external flow is increased, as shown on Fig. 2(b). When the external flow velocity becomes sufficiently high compared to the streaming velocity at rest, the two downstream vortices disappear and only the upstream vortices remain [see Fig. 2(c)].

Interestingly, some of the external streamlines that go through the bubble pair pass in the vicinity of the downstream bubble [see dark trajectory on Fig. 2(a)]. Such streamlines can be used to trap large particles in the downstream vortices, following a process similar to that already described in Ref. [13]. Wang et al. state that when the distance between the streamline followed by the particle and the bubble surface is smaller than the particle radius, the particle is deviated to the inside of the closed vortex. Thus, large particles will remain trapped until a saturation of particles occurs in the vortex area or until the acoustic excitation is ceased.

Upstream vortices can find utility in another way as they impose an exclusion zone. Indeed, no external streamline is passing in the vicinity of the upstream bubble and only particles already present in this region before the acoustic excitation remain trapped in these upstream vortices during the excitation period. As a consequence, the composition of the main flow can be modified independently of these exclusion zones, and the trapped fluid or particles later released, in order to control mixing conditions. Interestingly, this exclusion zone is reminiscent of the magnetosphere deviating the solar wind away from the earth [14] in which the magnetic field lines are curved by the solar wind, while a trapping zone appears around the earth. However, the mathematical expressions of the forces are too different to derive a simple analogy.
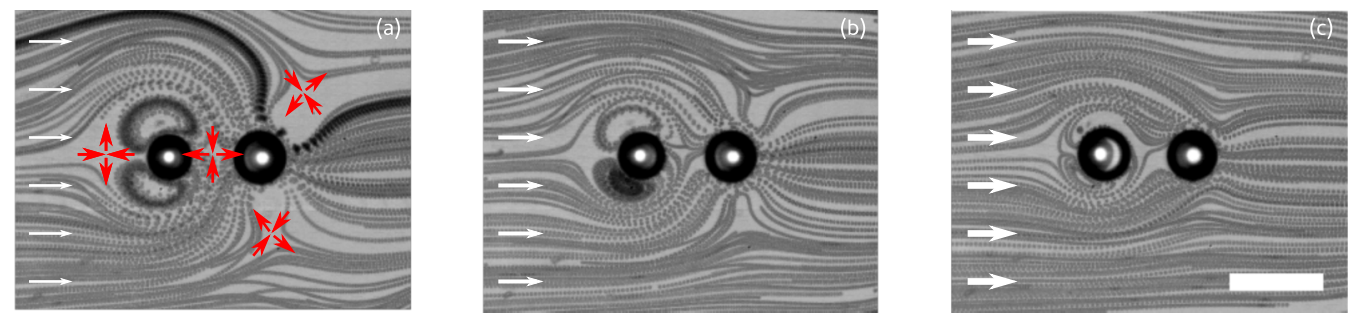

FIG. 2. Modification of the streaming pattern produced by two excited bubbles under an external flow. Flow is applied from the left with the following velocities: (a) $U_{\infty} / U_{\text {streaming }}=0.0874$, (b) $U_{\infty} / U_{\text {streaming }}=0.186$, and (c) $U_{\infty} / U_{\text {streaming }}=0.364$. The total flow is visualized using tracers $2 \mu \mathrm{m}$ in diameter. Their positions are superimposed to emphasize the streamlines. The same bubble pair was used over all three experiments, with constant acoustical pressure and frequency $(90 \mathrm{kHz})$. The scale bar corresponds to $100 \mu \mathrm{m}$ and the red arrows materialize the stagnation points. 


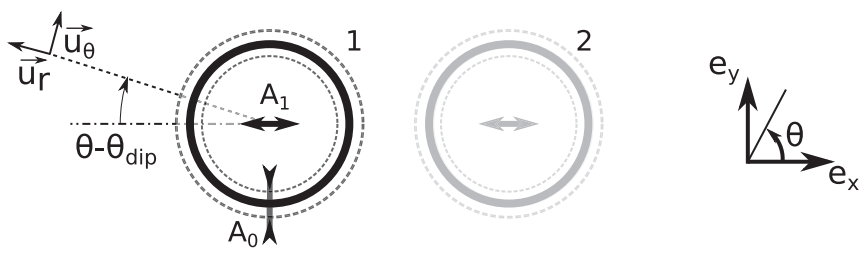

FIG. 3. Scheme of notations used in the theory. For a group of several bubbles (two bubbles here), the streaming is always calculated with respect to the generating bubble (here bubble 1). The notations are those used in this scheme. When $\theta_{\text {dip }}=\pi$, a fountain-type dipolar field is created.

\section{Theory for the flow field}

Acoustic streaming. In a previous article [9], we have shown that the breathing mode of one bubble acts as an excitation source for the translation mode of the neighboring bubble. This excitation is mediated by the Rayleigh waves emitted by the pulsating bubble and propagating in the PDMS upper and lower walls [15]. In the case of a bubble pair, each bubble combines its own breathing oscillation (mode 0 ) and the translation oscillation (mode 1) induced by its neighbor. The resulting oscillation leads to the generation of a streaming pattern whenever these two modes become out of phase.

Considering one bubble of the pair, we could describe the pulsation of its radius $R$ by introducing the parameters $\left(A_{0}, \phi_{0}\right)$ for the amplitude and phase of the breathing mode relative to the external excitation, so that $R=R_{0}+A_{0} \cos \left(\omega t+\phi_{0}\right)$ with $\omega$ being the excitation frequency and $R_{0}$ being the radius at rest. Similarly, we wrote $\left(A_{1}, \phi_{1}\right)$ for the amplitude and phase of mode 1 , such that the bubble center position evolved as $\mathbf{x}_{\text {center }}=\mathbf{A}_{1} \cos \left(\omega t+\phi_{1}\right)$. These notations allowed us to describe the acoustic streaming generated in the surrounding fluid. The streaming function is written as

$$
\Psi^{\mathrm{S}}=\frac{1}{2} A_{1} A_{0} \omega \sin \left(\phi_{0}-\phi_{1}\right)\left[\left(\frac{R_{0}}{r}\right)-\left(\frac{R_{0}}{r}\right)^{3}\right] \sin \left(\theta-\theta_{\text {dip }}\right),
$$

where $r$ is the distance from the bubble and $\theta_{\text {dip }}$ (also noted as $\theta_{1}$ in the simple case of a bubble pair) is the orientation of the translation mode with respect to the $x$ axis, pointing away from the second bubble (and in the direction $\theta_{\text {dip }}=\pi$ from the left bubble on Fig. 3).

The corresponding flow field $\mathbf{u}^{\mathrm{S}}(r, \theta)$, with components $u_{r}=\frac{1}{r} \frac{\partial \Psi}{\partial \theta}, u_{\theta}=-\frac{\partial \Psi}{\partial r}$, can be represented as

$$
\begin{aligned}
& u_{r}^{\mathrm{S}}=U_{\text {streaming }}\left[\left(\frac{R_{0}}{r}\right)^{2}-\left(\frac{R_{0}}{r}\right)^{4}\right] \cos \left(\theta-\theta_{\text {dip }}\right), \\
& u_{\theta}^{\mathrm{S}}=U_{\text {streaming }}\left[\left(\frac{R_{0}}{r}\right)^{2}-3\left(\frac{R_{0}}{r}\right)^{4}\right] \sin \left(\theta-\theta_{\text {dip }}\right),
\end{aligned}
$$

where the maximum streaming velocity $U_{\text {streaming }}$ is given by

$$
U_{\text {streaming }}=\frac{1}{2} \frac{A_{1} A_{0}}{R_{0}} \omega \sin \left(\phi_{0}-\phi_{1}\right)
$$

Note that the quantitative influence of surface Rayleigh waves on the streaming is neglected here. With surface waves, the streaming is increased for the same applied pressure amplitude [16] while the streaming patterns are similar. As a first approximation, we keep the no-surface wave approximation for the flow pattern and use $U_{\text {streaming }}$ as a fitting parameter. Importantly, we also remark that the flow velocity at the bubble surface has a vanishing $u_{r}^{\mathrm{S}}$ component but also a nonvanishing $u_{\theta}^{\mathrm{S}}$ component, since this flow is to be understood as the flow generated just outside of the thin acoustic oscillating boundary layer.

Hydrodynamic flow. In the case of an external flow (without acoustic streaming) applied along the $x$ axis with a velocity $U_{\infty}$, the hydrodynamic flow field around the bubble is the addition of a constant 

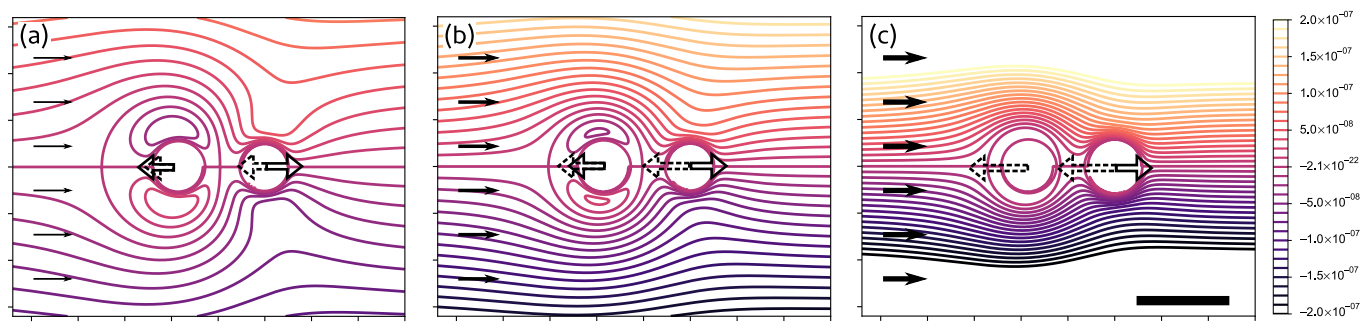

FIG. 4. Influence of an external flow on the streaming pattern produced by two bubbles. Plain hollow arrows represent the equivalent hydrodynamic dipoles induced by the streaming and dashed arrows represent the hydrodynamic dipoles induced on the bubbles by the external flow (arrows not at the same scale). Flow is coming from the left with the same ratio of external flow to streaming velocity than Fig. 2: (a) $U_{\infty} / U_{\text {streaming }}=0.0874$, (b) $U_{\infty} / U_{\text {streaming }}=0.186$, and (c) $U_{\infty} / U_{\text {streaming }}=0.364$. These values were chosen with the external flow values of Fig. 2 and assuming $U_{\text {streaming }}=6.18 \mathrm{~mm} / \mathrm{s}$. The scale bar is $100 \mu \mathrm{m}$ and the color bar represents values of isolines of the stream function in $\mathrm{m}^{2} \mathrm{~s}^{-1}$.

velocity and an induced flow field $\mathbf{u}^{\text {flow }}=\mathbf{u}^{\text {flow, } \infty}+\mathbf{u}^{\text {flow,induced }}$. In terms of stream functions, they are written as $\Psi^{\text {flow }}=\Psi^{\text {flow, } \infty}+\Psi^{\text {flow,induced }}$ with

$$
\Psi^{\text {flow, } \infty}=U_{\infty} r \sin (\theta)
$$

and with the dipolar field induced by the hydrodynamic interaction of incoming flow with the bubble

$$
\Psi^{\text {flow,induced }}=-U_{\infty} \frac{R_{0}^{2}}{r} \sin (\theta) .
$$

This translates in the following velocity fields:

$$
\begin{aligned}
& u_{r}^{\text {flow }, \infty}=U_{\infty} \cos (\theta), \\
& u_{\theta}^{\text {flow }, \infty}=-U_{\infty} \sin (\theta),
\end{aligned}
$$

and

$$
\begin{aligned}
& u_{r}^{\text {flow,induced }}=U_{\infty}\left[-\left(\frac{R_{0}}{r}\right)^{2}\right] \cos (\theta), \\
& u_{\theta}^{\text {flow,induced }}=U_{\infty}\left[-\left(\frac{R_{0}}{r}\right)^{2}\right] \sin (\theta),
\end{aligned}
$$

meaning a dipole pointing in the opposite direction of the flow. As a result, the velocity on the bubble surface is equal to zero, respecting a no-slip boundary condition.

Total flow. If we assume the streaming intensity is not affected by this external flow, which seems to be a good approximation as long as the bubble surface does not present Faraday instability [17], the total flow field is found by the addition of the previous flow fields:

$$
\mathbf{u}=\mathbf{u}^{\text {flow, } \infty}+\sum_{\text {bubbles }} \mathbf{u}^{\text {flow,induced }}+\sum_{\text {bubbles }} \mathbf{u}^{\mathrm{S}} .
$$

This approximation is not strictly valid near the bubble surface, since the induced and streaming flows created by one bubble will make a small contribution at the bubble surface of any other bubble, slightly disturbing the no-crossing velocity condition. For this reason, the following simulations have been performed by using the stream function and not the velocity field. Furthermore, we enforced a no-crossing velocity to each bubble by forcing the value of the stream function to a constant on the bubble surface. Streamlines were obtained by plotting the stream function isovalues. 

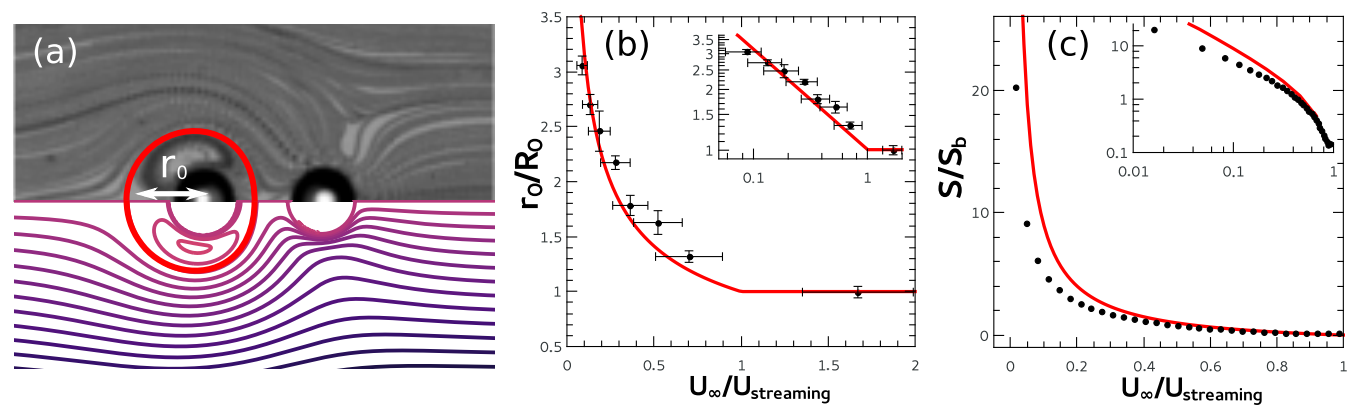

FIG. 5. (a) Experimental exclusion zone superimposed with theoretical prediction from Ref. [9]. The excluded zone is the zone within the red curve. The external flow is coming from the left. (b) Experimental distance of the "stop point" as a function of the external flow. Curve is a fit using Eq. (7), giving $U_{\text {streaming }}=$ $6.18 \mathrm{~mm} / \mathrm{s}$. Errors bars for $U_{\infty}$ are the standard deviation of the speed of particles outside the streaming flow. Errors bars for $r / R_{0}$ take origin in the drift of the bubbles' radii during the experiment. All points were acquired during the same experiment, varying the external flow $U_{\infty}$ from 10 to $0.5 \mathrm{~mm} / \mathrm{s}$. The excitation frequency is $90 \mathrm{kHz}$. (c) Numerical excluded surface, normalized by the bubble surface: Black dots are the results of numerical simulations whereas the red line is the excluded surface in the case of a circular excluded zone with the radius given by Eq. (7).

Figure 4 shows the streaming figure created by two bubbles with an external flow, where the streaming velocity $U_{\text {streaming }}=6.18 \mathrm{~mm} / \mathrm{s}$ was adjusted in order to reproduce the experimental findings of Fig. 2, in particular the position of the upstream stagnation point. We recover the upstream exclusion zones observed experimentally. Thus, the combination of the external flow with the streaming flow is sufficient to understand the emergence of these regions.

\section{Size of the exclusion zone}

As could be seen in Fig. 4, the exclusion zone is delimited upstream from the bubble pair by a stagnation point located on the axis. This stagnation point is the consequence of a streaming velocity directed against the flow in the fountain mode $\left(U_{\text {streaming }}>0\right)$. If we neglect the contributions from the second bubble, the position $r_{0}$ of this point located on $\theta=\pi$ is given by $u_{r}\left(r=r_{0}, \theta=\pi, \theta_{\mathrm{dip}}=\right.$ $\pi)=0$, with $r_{0}$ such that

$$
U_{\text {streaming }}\left[\left(\frac{R_{0}}{r_{0}}\right)^{2}-\left(\frac{R_{0}}{r_{0}}\right)^{4}\right]-U_{\infty}\left[1-\left(\frac{R_{0}}{r_{0}}\right)^{2}\right]=0,
$$

which gives only one solution satisfying $\frac{r_{0}}{R_{0}}>1$,

$$
\frac{r_{0}}{R_{0}}=\sqrt{\frac{U_{\text {streaming }}}{U_{\infty}}} .
$$

Experimentally, the parameter $r_{0}$ is determined by selecting the minimum value of each pixel in a given movie, thus highlighting the tracers trajectory, and then measuring the distance between the center of the bubble and the stagnation point as shown in Fig. 5(a). Experimental values of $r_{0} / R_{0}$ are in good agreement with Eq. (7) as can be seen in Fig. 5(b).

As $U_{\text {streaming }}$ is function of the square of the acoustic pressure (the amplitudes $A_{0}$ and $A_{1}$ being proportional to the acoustic pressure), it is theoretically possible to widen the exclusion zone area by increasing the acoustic pressure. The first limitation here is to get a sufficient anchor strength to prevent the bubbles to escape the capillary trap.

As observed in Figs. 2(a) and 5(a), the exclusion zone is not circular. Its deviation to a circular form can be estimated by numerical simulations. The exclusion area is determined by numerically 

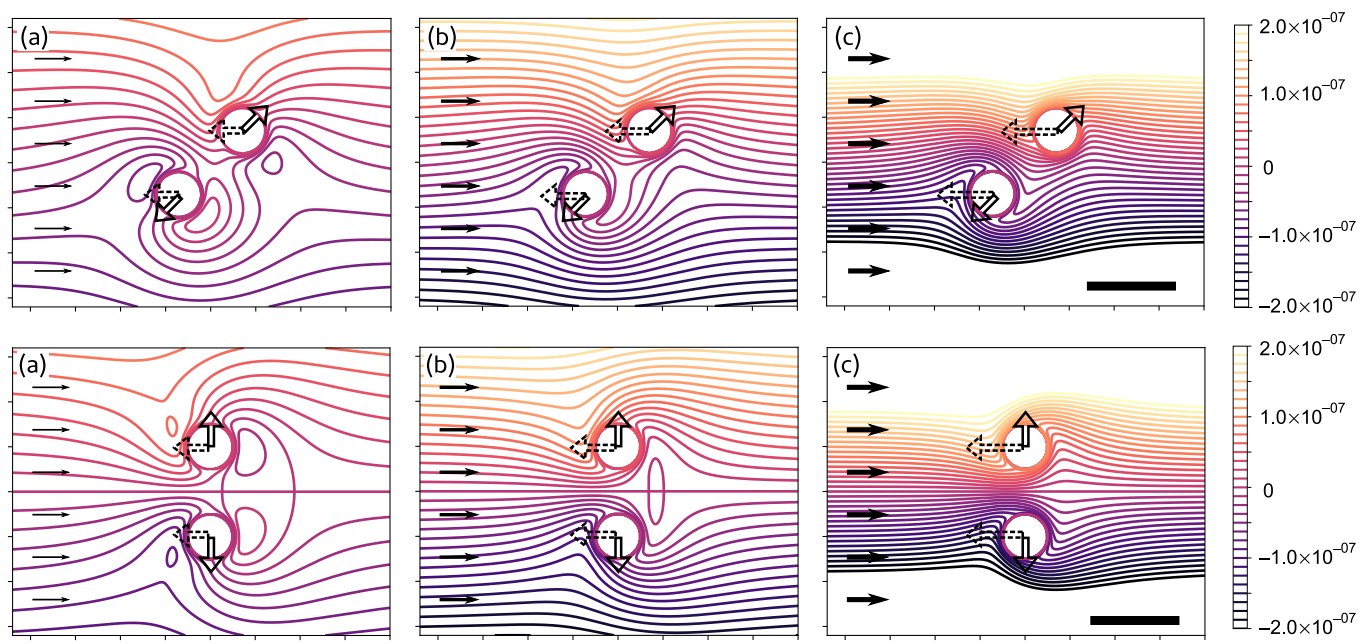

FIG. 6. Influence of an increasing external flow on the streaming pattern produced by two bubbles oriented at 45 and $90^{\circ}$. Same ratios of external flow to streaming velocity in Figs. 2 and 4: (a) $U_{\infty} / U_{\text {streaming }}=0.0874$, (b) 0.186 , and (c) 0.364 . Dashed arrows represent the dipole induced for each bubble by the external flow whereas plain arrows represent dipoles induced by the acoustic excitation. The scale bar is $100 \mu \mathrm{m}$ and the color bar represents values of isolines of the stream function in $\mathrm{m}^{2} \mathrm{~s}^{-1}$.

integrating the area inside the zero value streamline [see Fig. 5(c)] and can be compared to the area given by a circular exclusion zone of theoretical radius $r_{0}$ given by Eq. (7).

\section{B. Role of dipole orientation}

In the previous part, the axis of the bubble pair was exactly aligned with the flow. We now consider the situations where the axis joining the bubble pair is not aligned with the main flow. Our simulations show that as soon as the axis is inclined, the exclusion zones separate in two distinct zones, with the one downstream of the first bubble being larger; see Figs. 6(a)-6(c). These zones shrink when the external flow velocity increases. Interestingly, as some of the streamlines are passing in the vicinity of the upstream bubble, while going through the channel, the trapping process takes place: Large particles in such a flow would get trapped in the vortices of the upstream bubble, as long as they are on the same latitude as the bubble.

At a $90^{\circ}$ angle (Fig. 6 bottom), bubble interactions generates one large vortex downstream of each bubble. We recover the same type of flow as described in Wang et al. [18]. In fact, the particular case of a hemicylindrical bubble anchored on a wall can be explained as the result of the interaction between two cylindrical bubbles staying at the same position in the channel and oscillating out of phase. By introducing a distance $D$ between both bubbles of the pair, new streamlines appear: They pass in between the two bubbles and are then forced to go in the vicinity of the bubble. Thus, large particles which latitude stays in between the two bubbles will get trapped in these two large containers downstream of each bubble. These zones are no more exclusion zones as in the case of the anchored hemicylindrical bubble, but they are trapping zones.

\section{TOWARD A LARGE GROUP OF BUBBLES}

We now extend the previous two-body interaction to many bubbles. For an assembly of bubbles as considered in the present study, the description used earlier for a bubble pair is still valid. However, we need to know the amplitude of their pulsation and translation oscillation.

When there is more than one neighbor around a bubble of interest (labeled with index $i$ ), each of the neighbors (now labeled with index $j$ ) would induce, in the absence of the others, a translation 
of the bubble described by a complex vector $\overline{\mathbf{A}_{1}^{j \rightarrow i}}$ aligned with the axis linking the bubble with neighbor $j$, such that the center of the bubble of interest pulsates as $\mathbf{x}_{\text {center }}^{j \rightarrow i}=\mathbf{A}_{1}^{j \rightarrow i} \cos \left(\omega t+\phi_{1}^{j \rightarrow i}\right)=$ $\operatorname{Re}\left[\overline{\mathbf{A}_{1}^{j \rightarrow i}} \exp (i \omega t)\right]$ because of the influence of bubble $j$.

Acoustic streaming flows are mainly the results of mixed-mode oscillations: a volume oscillations of complex amplitude $\overline{A_{0}}$, such that the radius pulsates as $R=R_{0}+A_{0} \cos \left(\omega t+\phi_{0}\right)=R_{0}+$ $\operatorname{Re}\left[\overline{A_{0}} \exp (i \omega t)\right]$, and translation oscillations described above.

For a pair of bubbles, the streaming velocity around a bubble is described by a dipolar-like flow field where the dipole amplitude and direction are given by the vector

$$
\mathbf{U}_{\text {streaming }}^{j \rightarrow i}=A_{0}{ }^{i} \mathbf{A}_{1}^{j \rightarrow i} \sin \left(\phi_{0}-\phi_{1}^{j}\right) \omega / 2 R_{0}=\operatorname{Re}\left[i \overline{A_{0}{ }^{i}} \overline{\mathbf{A}_{1}^{j}}\right] \omega / 2 R_{0},
$$

if we adopt the complex notation.

In the linear acoustic approximation, the bubble center submitted to the action of all the several neighbor bubbles is expected to translate with an amplitude set by the sum of these amplitudes, such as $\mathbf{x}_{\text {center }}=\operatorname{Re}\left[\overline{\mathbf{A}_{1}} \exp (i \omega t)\right]$ with $\overline{\mathbf{A}_{1}}=\sum_{j} \overline{\mathbf{A}_{1}^{j}}$. For more than one neighbor the translation is set by a sum of translations vectors, and we therefore expect the streaming around a bubble to be given by

$$
\mathbf{U}_{\text {streaming }}=\operatorname{Re}\left[i \overline{A_{0} i}\left(\sum_{j} \overline{\mathbf{A}_{1}^{j}}\right)\right] \omega / 2 R_{0}=\sum_{j} \mathbf{U}_{\text {streaming }}^{j} .
$$

We feel therefore confident in adding up the streaming contributions from each bubble pair, each described by a dipole $\mathbf{U}_{\text {streaming }}^{j}$ at long distances.

\section{A. Determination of the oscillations amplitudes}

For these cases, the different bubbles are not equivalent anymore, and thus the values of the parameters $A_{0}$ and $A_{1}^{j}$ for each bubble are still to be found. To do so, we use the theory developed by Mekki-Berrada et al. [9], which can be adapted for the $N$-bubble case. A bubble $i$ in an assembly of $N$ bubbles will pulsate with an amplitude $\overline{A_{0}^{i}}$ thanks to both the external acoustic excitation $P_{a c}$ and the Rayleigh waves propagating in the top and bottom walls, produced by any other bubble $j$. These Rayleigh waves will also induce the translation mode $\overline{A_{1}^{j \rightarrow i}}$ on the bubble $i$. It has been shown [9] that in the case of a bubble $i$, in the vicinity of another bubble $j$, these two parameters can be calculated as

$$
\begin{gathered}
\overline{A_{0}^{i}}\left[\alpha u\left(R_{i}, R_{i}\right)-2 \frac{\kappa p_{0}}{R_{i}}\right]+\alpha u\left(D_{i, j}, R_{j}\right) \overline{A_{0}^{j}}=\overline{P_{a c}}, \\
\overline{A_{1}^{j \rightarrow i}}\left[\alpha v\left(R_{i}\right)\right]-\alpha w\left(D_{i, j}, R_{j}\right) \overline{A_{0}^{j}}=0,
\end{gathered}
$$

with

$$
\begin{cases}u\left(D_{1}, D_{2}\right)= & \frac{H_{0}^{(2)}\left(k D_{1}\right)}{H_{1}^{(2)}\left(k D_{2}\right)} \\ v(R)= & \frac{H_{1}^{(2)}(k R)}{H_{1}^{(2)}(k R)} \\ w\left(D_{1}, D_{2}\right)= & k D_{2} \frac{H_{1}^{(2)}\left(k D_{1}\right)}{H_{1}^{(2)}\left(k D_{2}\right)} \\ \alpha= & \frac{\rho \omega^{2}}{k}\end{cases}
$$

where $k=\omega / c_{R}$ is the Rayleigh wave number, $c_{r} \approx 40 \mathrm{~m} / \mathrm{s}$ is the velocity of the Rayleigh wave at the channel wall interface, $\rho$ is the liquid density (1 in the current case), $R_{i}$ is the radius of bubble $i$, $D_{i, j}$ is the distance separating bubbles $i$ and $j, p_{0}$ is the average pressure around the bubbles which is $1.01 \times 10^{5} \mathrm{~Pa}, \kappa$ is the polytropic index of the gas which is around 1.4 for air, $H_{n}^{(2)}$ is the Hankel function of the second kind of order $n$, and $H_{n}^{\prime(2)}$ is its derivative. 

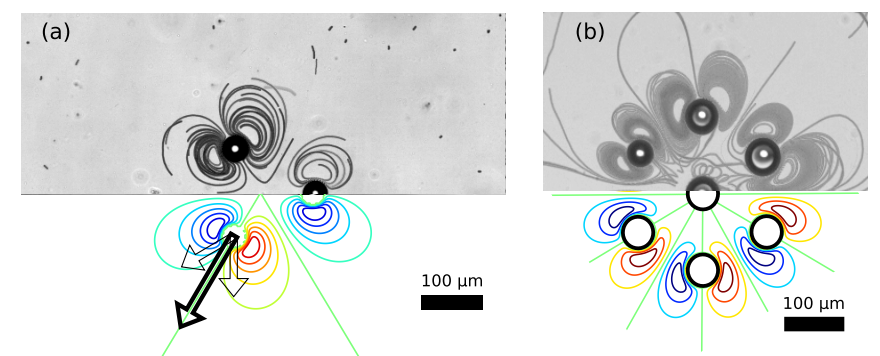

FIG. 7. Comparison of the model with experiments for more than two bubbles in the absence of external flow. The top images are experimental results and the bottom images are numerical results according to the theory developed at the beginning of this section. (a) Three bubbles under ultrasonic excitation without external flow (excitation frequency is $91 \mathrm{kHz}$ ). The bold plain arrow represents the total streaming dipole for one bubble, which can be decomposed in the two dipoles induced by each neighbors represented by the thin plain arrows. (b) Seven bubbles under ultrasonic excitation (excitation frequency is $53 \mathrm{kHz}$ ).

Equations (9) and (10) can be further extended to an assembly of $N$ bubbles, leading to

$$
\begin{gathered}
\overline{A_{0}^{i}}\left[\alpha u\left(R_{i}, R_{i}\right)-2 \frac{\kappa p_{0}}{R_{i}}\right]+\alpha \sum_{j \neq i} u\left(D_{i, j}, R_{j}\right) \overline{A_{0}^{j}}=\overline{P_{a c}}, \\
\overline{A_{1}^{j \rightarrow i}}=\frac{\alpha w\left(D_{i, j}, R_{j}\right) \overline{A_{0}^{j}}}{\left[\alpha v\left(R_{i}\right)\right]} .
\end{gathered}
$$

This linear system of equations can be solved in order to find $\overline{A_{0}^{i}}$ and $\overline{A_{1}^{j \rightarrow i}}$ for each bubble. In practice, the sum over all the bubbles $j$ present in the system, apart from the considered bubble $i$, can be reduced to a sum over the neighbors of the bubble $i$. This simplifies the resolution of the system without substantially affecting the results as the amplitude of the Rayleigh waves decreases very fast with the distance [see Eq. (10)].

\section{B. Validity of the model in the absence of external flow}

If coherent with the dipolar approximation developed in the previous section, the streaming pattern produced by a greater number $N$ of bubbles should show $2 N$ vortices without any external flux. As can be seen in Fig. 7, this relation is still verified for a group of three and seven bubbles; moreover, the direction of streaming is in agreement with the linear addition of two dipoles induced by the neighbors for these two cases (see Fig. 7, left).

In the case of a regular lattice of seven bubbles, one can notice that the experimental streaming flow close to the center bubble slightly deviates from the theoretical model. Because of the lattice symmetry, the translation mode of the center bubble is theoretically supposed to be zero, but higher modes can still interact with the volume pulsation and lead to close recirculations in the vicinity of the center bubble. In parallel, the deformation of the PDMS walls caused by the volume pulsation can also contribute to low-intensity three-dimensional (3D) streaming effects.

\section{Influence of an external flow on a greater number of bubbles}

One can now study if the theory developed in this section for a number of bubbles greater than two is still valid with the addition of an external flow. We show in Fig. 8 a comparison between experimental and simulated streaming flows around three bubbles undergoing an external flow. This figure shows that the theory developed at the beginning of this section is still in clear agreement with experimental data for more than two bubbles. For this case, there is not a clearly defined excluded zone as there was when dealing with the two-bubble case; however, one can see that six smaller excluded 

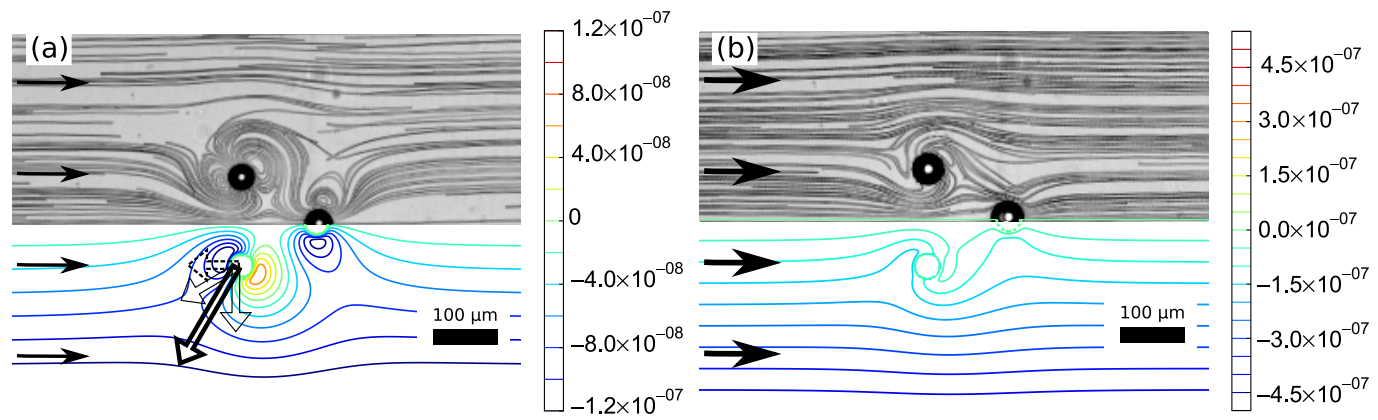

FIG. 8. Streaming around a group of three bubbles undergoing an external flow: comparison between experiments (top) and the model represented in this section. The external flow is applied from the left with the following velocities: (a) $U_{\infty}=0.295 \mathrm{~mm} / \mathrm{s}$ and (b) $U_{\infty}=1.474 \mathrm{~mm} / \mathrm{s}$. The simulation was fitted to the experiments by adjusting the streaming velocities: for (a) $U_{\text {streaming }}=8.4 \mathrm{~mm} / \mathrm{s}$ and for (c) $U_{\text {streaming }}=$ $5.6 \mathrm{~mm} / \mathrm{s}$. In addition to the dipolar flows already present in Fig. 7, there is a dipolar flow produced by the interaction between the external flow and the bubbles represented by the dashed arrow. Excitation frequency is $89 \mathrm{kHz}$.

zones appear at the vicinity of the three bubbles. Just like the two-bubble case, these excluded zones shrink with the increase of the external flow until they finally disappear, as can be seen in Fig. 8(b).

\section{Pinball}

This approach can be generalized to a very large number of bubbles. In Fig. 9(a), we show an experiment featuring the complex streaming around many bubbles $(N=59)$, showing that each tracer particle enters in a microfluidic "pinball." Pairs of vortices can be recognized, such as around the bottom-most right bubble, but the combination of the flow and the streaming results in very complex patterns. For a large and regular array of bubbles, symmetry should impose no or little flow in the middle of the array, as is the case in Fig. 7(b). Here, the presence of defects in the lattice leads to the development of vortices inside the array. The prediction of the streamlines by the model presented in this article (Fig. 9) is not as good as it was for a lesser number of bubbles. This is caused by the fact the additivity of the streamlines is a less good approximation for a greater number of bubbles, thus showing its limit in this case.
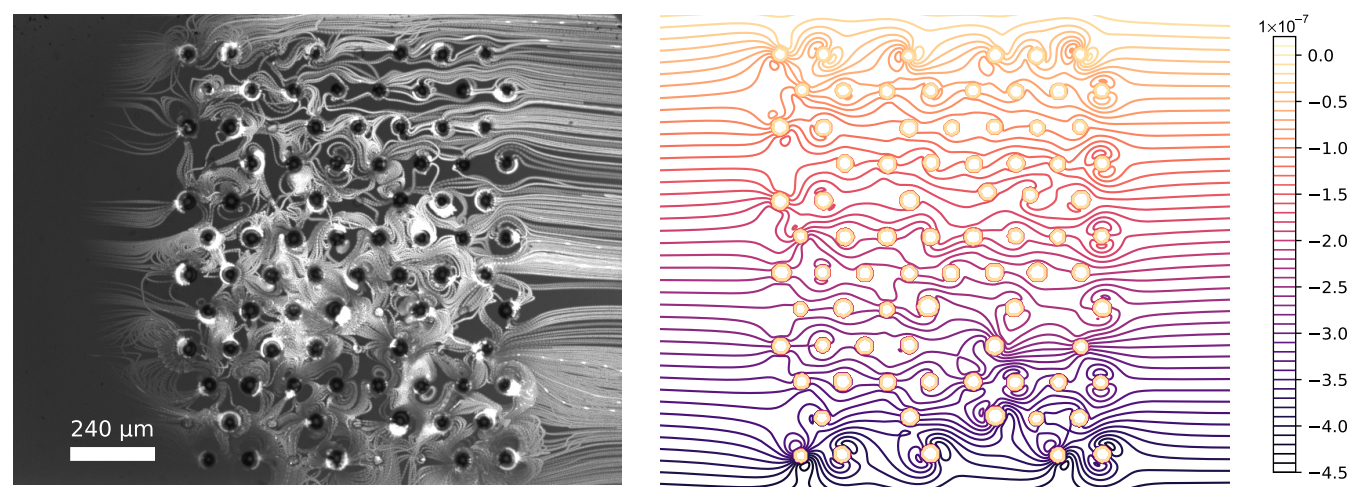

FIG. 9. Left: A "pinball" of microfluidic bubbles. The flow is coming from the right with a velocity $U_{\infty}=$ $304 \mu \mathrm{m} / \mathrm{s}$. Right: Model described in Sec. IV A, using the acoustic pressure amplitude $P_{a c}$ as the only adjusting parameter, showing qualitative agreement with the experiment. 


\section{CONCLUSION AND OUTLOOK}

In conclusion, we have shown how closed recirculation zones appear around oscillating bubbles when submitted to an external flow. These zones are upstream in the case of two bubbles aligned with the flow and act as exclusion zones. Their size decreases with applied external flows. The recirculation zones are located downstream when bubbles are perpendicular to the flow; because of the spacing between the two bubbles, these zones can act as trapping zones.

The model developed here for the streaming can be extended to a large number of interacting bubbles, with a linear superposition of dipolar-like streaming flows, each dipole being set by pairwise interactions. Even if the linear superposition shows its limit for a very large group of bubbles, it shows very good agreement for smaller groups.

The recirculation zones will prove useful to design applications where it is desired to isolate a part of the flow (to obtain chemical reactions). Another natural application will be to enhance mixing by having zones repeatedly stirred and advected with sequences of transient insonations under flow, as an alternative process to the design presented in Ref. [19]. Last, it can be seen that recirculation zones tend to catch particles, which will be the subject of further studies.

\section{ACKNOWLEDGMENTS}

This research has received funding from the European Research Council under the European Union's Seventh Framework Programme (FP7/2007-2013)/ERC Grant Agreement No. 614655 "Bubbleboost."

[1] X. Ding, S.-C. S. Lin, B. Kiraly, H. Yue, S. Li, I.-K. Chiang, J. Shi, S. J. Benkovic, and T. J. Huang, On-chip manipulation of single microparticles, cells, and organisms using surface acoustic waves, Proc. Natl. Acad. Sci. USA 109, 11105 (2012).

[2] S. B. Q. Tran, P. Marmottant, and P. Thibault, Fast acoustic tweezers for the two-dimensional manipulation of individual particles in microfluidic channels, Appl. Phys. Lett. 101, 114103 (2012).

[3] J. Lighthill, Acoustic streaming, J. Sound Vibr. 61, 391 (1978).

[4] P. Marmottant and S. Hilgenfeldt, Controlled vesicle deformation and lysis by single oscillating bubbles, Nature (London) 423, 153 (2003).

[5] A. A. Doinikov and A. Bouakaz, Acoustic microstreaming around a gas bubble, J. Acoust. Soc. Am. 127, 703 (2010).

[6] B. Rallabandi, C. Wang, and S. Hilgenfeldt, Two-dimensional streaming flows driven by sessile semicylindrical microbubbles, J. Fluid Mech. 739, 57 (2014).

[7] D. Ahmed, X. Mao, J. Shi, B. K. Juluri, and T. J. Huang, A millisecond micromixer via single-bubble-based acoustic streaming, Lab. Chip 9, 2738 (2009).

[8] R. Dangla, S. Lee, and C. N. Baroud, Trapping Microfluidic Drops in Wells of Surface Energy, Phys. Rev. Lett. 107, 124501 (2011).

[9] F. Mekki-Berrada, T. Combriat, P. Thibault, and P. Marmottant, Interactions enhance the acoustic streaming around flattened microfluidic bubbles, J. Fluid Mech. 797, 851 (2016).

[10] B. Rallabandi, A. Marin, M. Rossi, C. J. Kähler, and S. Hilgenfeldt, Three-dimensional streaming flow in confined geometries, J. Fluid Mech. 777, 408 (2015).

[11] D. C. Duffy, J. C. McDonald, O. J. A. Schueller, and G. M. Whitesides, Rapid prototyping of microfluidic systems in poly(dimethylsiloxane), Anal. Chem. 70, 4974 (1998).

[12] A Fast And Simple Tracker, http://www-liphy.ujf-grenoble.fr/Thomas-Combriat-en?lang=en.

[13] C. Wang, S. V. Jalikop, and S. Hilgenfeldt, Size-sensitive sorting of microparticles through control of flow geometry, Appl. Phys. Lett. 99, 034101 (2011).

[14] J. G. Lyon, The solar wind-magnetosphere-ionosphere system, Science 288, 1987 (2000). 
[15] D. Rabaud, P. Thibault, M. Mathieu, and P. Marmottant, Acoustically Bound Microfluidic Bubble Crystals, Phys. Rev. Lett. 106, 134501 (2011).

[16] A. A. Doinikov, T. Combriat, P. Thibault, and P. Marmottant, Acoustic streaming produced by a cylindrical bubble undergoing volume and translational oscillations in a microfluidic channel, Phys. Rev. E 94, 033109 (2016).

[17] R. Dangla and C. Poulain, When sound slows down bubbles, Phys. Fluids 22, 041703 (2010).

[18] C. Wang, S. V. Jalikop, and S. Hilgenfeldt, Efficient manipulation of microparticles in bubble streaming flows, Biomicrofluidics 6, 012801 (2012).

[19] C. Neils, Z. Tyree, B. Finlayson, and A. Folch, Combinatorial mixing of microfluidic streams, Lab. Chip 4, 342 (2004). 